This document is the accepted manuscript version of the following article:

Y. S. Ko, F. A. Nüesch, D. Damjanovic, D. M. Opris (2017), An All-Organic Elastomeric Electret Composite

Adv. Mater., 2017, 29(1), 1603813.

DOI: $10.1002 / \mathrm{adma} .201603813$

\title{
An All-Organic Elastomeric Electret Composite
}

By Yee Song Ko, ${ }^{*}$ Frank A. Nüesch, Dragan Damjanovic and Dorina M. Opris*

\section{Y. S. Ko, Prof. F. A. Nüesch, Dr. D. M. Opris}

Swiss Federal Laboratories for Materials Science and Technology Empa, Laboratory for

Functional Polymers, Überlandstr. 129, CH-8600, Dübendorf, Switzerland

E-mail: dorina.opris@empa.ch; Yeesong.Ko@empa.ch

Y. S. Ko, Prof. F. A. Nüesch, Prof. D. Damjanovic

École Polytechnique Fédérale de Lausanne (EPFL), Institut des matériaux, Station 12, CH 1015, Lausanne, Switzerland

Keywords: Electret, PDMS, Disperse Red 1, Piezoelectric, Elastomer

Poled polymer electrets with oriented molecular dipoles are known to exhibit piezoelectricity since the phenomenon was discovered in wax and rosin electrets. ${ }^{[1]}$ In 1969 piezoelectricity was found in poled poly(vinylidene fluoride) (PVDF) which has since lead to widespread application as electromechanical transducers and sensors due to their low weight, mechanical flexibility and toughness. ${ }^{[2-6]}$ Poling refers to the orienting of dipolar segments within the material using an electric field. While ferroelectric polymers such as PVDF can be poled above the glass transition temperature $\left(T_{g}\right)$, forming stable poled structures at room temperature, ${ }^{[7]}$ glassy dipole electrets, such as the aforementioned wax and rosin electret, or more topically poly(methyl methacrylate) ${ }^{[8]}$ require a thermal poling process. In this case the dipolar segments of the polymer are poled at elevated temperatures near $T_{g}$ and the overall polarization is frozen in when the material is cooled in a high electric field below $T_{g}{ }^{[9]}$ The polarization is compensated by charges on the electrodes and at phase boundaries, which help to stabilize the polarization. ${ }^{[10,11]}$ When the material is mechanically stressed and deformed, the change in polarization is accompanied by a flow of charge from one electrode to the other, (Figure 1). ${ }^{[12]}$ 


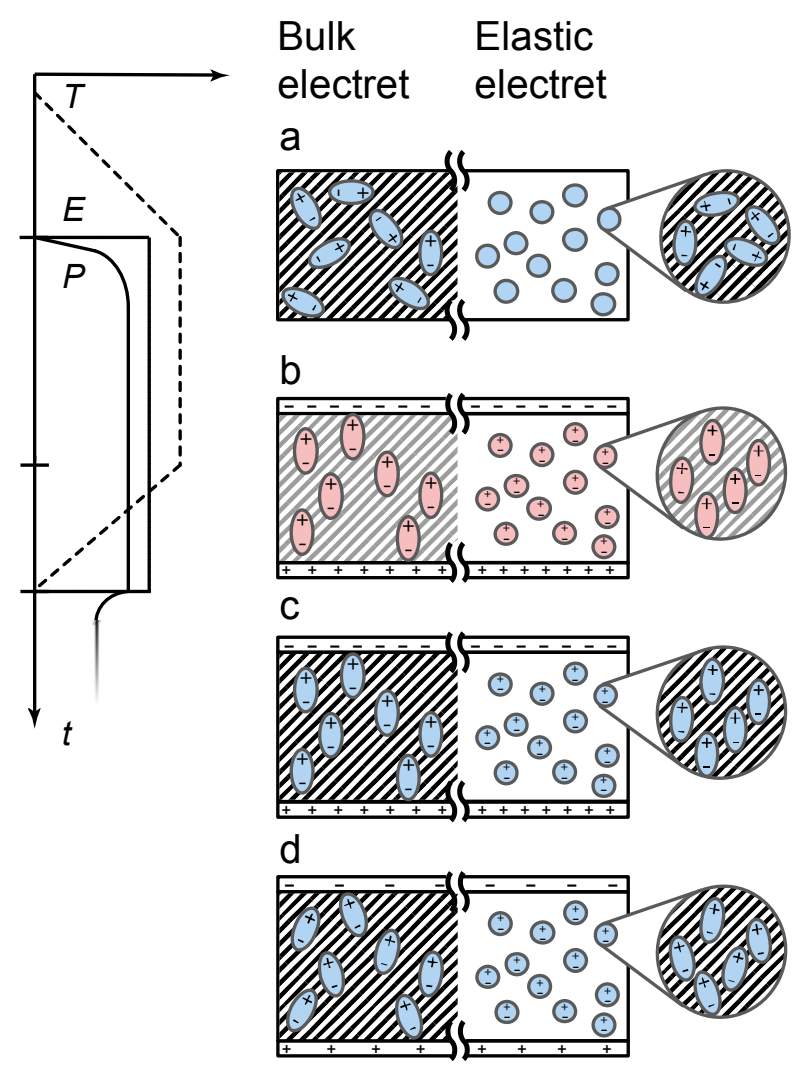

Figure 1. Poling procedure $(T=$ Temperature, $E=$ Electric field, $P=$ Polarization, $t=$ time $)$ and the corresponding schematic representation of the microstructure of a typical dipolelectret vs the proposed elastic electret composite. During the procedure the materials a) with randomly oriented dipoles are heated. b) Once the poling temperature is reached the dipoles are oriented in an electric field, and c) cooled while still under an electric field to obtain d) polarized electret materials. e) When under pressure, the dipole density of the materials change and a current flows.

The thermal poling of polymers with permanent molecular dipoles has been extensively used in the field of non-linear optical (NLO) polymers for the creation of non-centrosymmetric materials which have large stable polarization values. ${ }^{[13,14]}$ However, NLO polymers generally 
do not have high piezoelectric coefficient $\left(d_{33}\right)$. Elastomeric electrets would greatly expand the range of possible applications, such as wearable human activity monitoring, ${ }^{[15]}$ sensitive skin for robotics, ${ }^{[16]}$ large strain active sensors, ${ }^{[17]}$ or generators for stretchable electronics. ${ }^{[18]}$ This work exploits the high polarization of NLO materials for the synthesis of elastic electrets by blending NLO polymer particles into an elastic matrix. An elastic electret is eventually prepared, by poling such a composite. The comparison of a bulk (NLO)- and a composite electret is schematically shown side by side in Figure 1. Composite electrets were recently modelled more generally with a simple system comprising positive and negative charges, representing the dipoles, coupled by two different springs, representing the mechanical properties of the two phases. The resulting formula is intuitive and helpful in illustrating the source piezoelectricity in a composite material: ${ }^{[19]}$

$d_{33} \approx-\frac{P}{Y_{M}}+\frac{P}{Y_{D}}$

where $P$ is the remanent polarization of the composite and $Y_{M}$ and $Y_{D}$ are the elastic moduli of the matrix and the dipole respectively. The equation shows that the elastic moduli of the components should be different in order to generate a current. If the matrix is very $\operatorname{stiff}\left(Y_{M}\right.$ $>>Y_{D}$ ) the main contribution to $d_{33}$ comes from a lowered overall sample polarization after squeezing the charges together. Conversely, a stiff dipole material $\left(Y_{M}<<Y_{D}\right)$ will see an increased dipole density, because only the space between the dipoles is compressed. The mirrored effect on the sample polarization, and therefore the current direction, is the reason for the different sign in both components on the right side of Equation (1). In order to maximize the piezoelectric efficiency, the proposed system should therefore have a high polarization, which is provided by the NLO polymer, and low matrix elastic modulus, which cross-linked polydimethylsiloxane (PDMS) is well known for. The main barrier to such an approach is the production of polymeric nanoparticles, which tends to be rather challenging. However, recent developments in nanoprecipitation show that in theory any kind of polymer can be obtained in particle form. ${ }^{[20]}$ This was indeed accomplished for a NLO material in this 
work, enabling the production of an electret composite with poled dipoles. A standard material in the NLO field, poly(methyl methacrylate-co-Disperse Red 1 Methacrylate) (PMMA-co-DR1MA) is a copolymer composed of poly(methyl methacrylate) (PMMA) and a methacrylate modified with a side-group of 4-[ethyl (2-hydroxyethyl) amino]-4-nitrobenzene, usually called Disperse Red 1 (DR1). ${ }^{[21]}$ The molecular structure can be seen in Figure $2 \mathbf{a}$. DR1 is known for its large dipole moment $\mu$ of $7.5-9.5 \mathrm{D}^{\left[{ }^{[2]}\right.}$ By incorporating this dipole as a side-chain in a polymer, the lifetime of the poled structure can be increased drastically. ${ }^{[23,24]}$ Fully functionalized DR1 side-chain structures were measured to have $d_{33}$ values up to $2 \mathrm{pCN}^{-}$ ${ }^{1}$ when poled. ${ }^{[25]}$ Some PMMA-co-DR1MA copolymers are commercially available and can also easily be synthesized to incorporate any concentration of DR1 (see SI). ${ }^{[26,27]}$ For this work a copolymer with 50 mol\% DR1 repeating units was chosen and used in all the subsequent steps.

As mentioned before, the nanoprecipitation method (Figure $2 \mathbf{b}$ ) allows for the formation of copolymer submicron particles. It is carried out by slowly adding an initial polymer solution to a non-solvent in which the polymer nucleates and aggregates until colloidal stability has been reached in what has been termed the "ouzo effect". ${ }^{[28]}$ It was possible to vary the size of the copolymer particles between $50 \mathrm{~nm}$ and $420 \mathrm{~nm}$, with attempts at larger diameters resulting in highly polydisperse suspensions. A high dilution is generally desirable to ensure the system remaining in the ouzo region, which is the concentration regime where nanoprecipitation can take place. This is the reason why higher concentrations, which would be needed to produce larger particles, are not successful. Once the particles have formed, they are collected by filtration via a membrane filter (Nylon, $0.45 \mu \mathrm{m}$ pore size). Small spherical particles with a diameter of $60 \mathrm{~nm}$ were produced as can be seen in the secondary electron microscopy (SEM) image (Figure 2d). The size distribution was measured with dynamic light scattering (DLS) and by measuring the sizes of 50 particles in the SEM image (Figure 2c). 
Both methods lead to overlapping size distributions. DLS analysis shows moderate polydispersity ${ }^{[29]}$ of 0.27 .
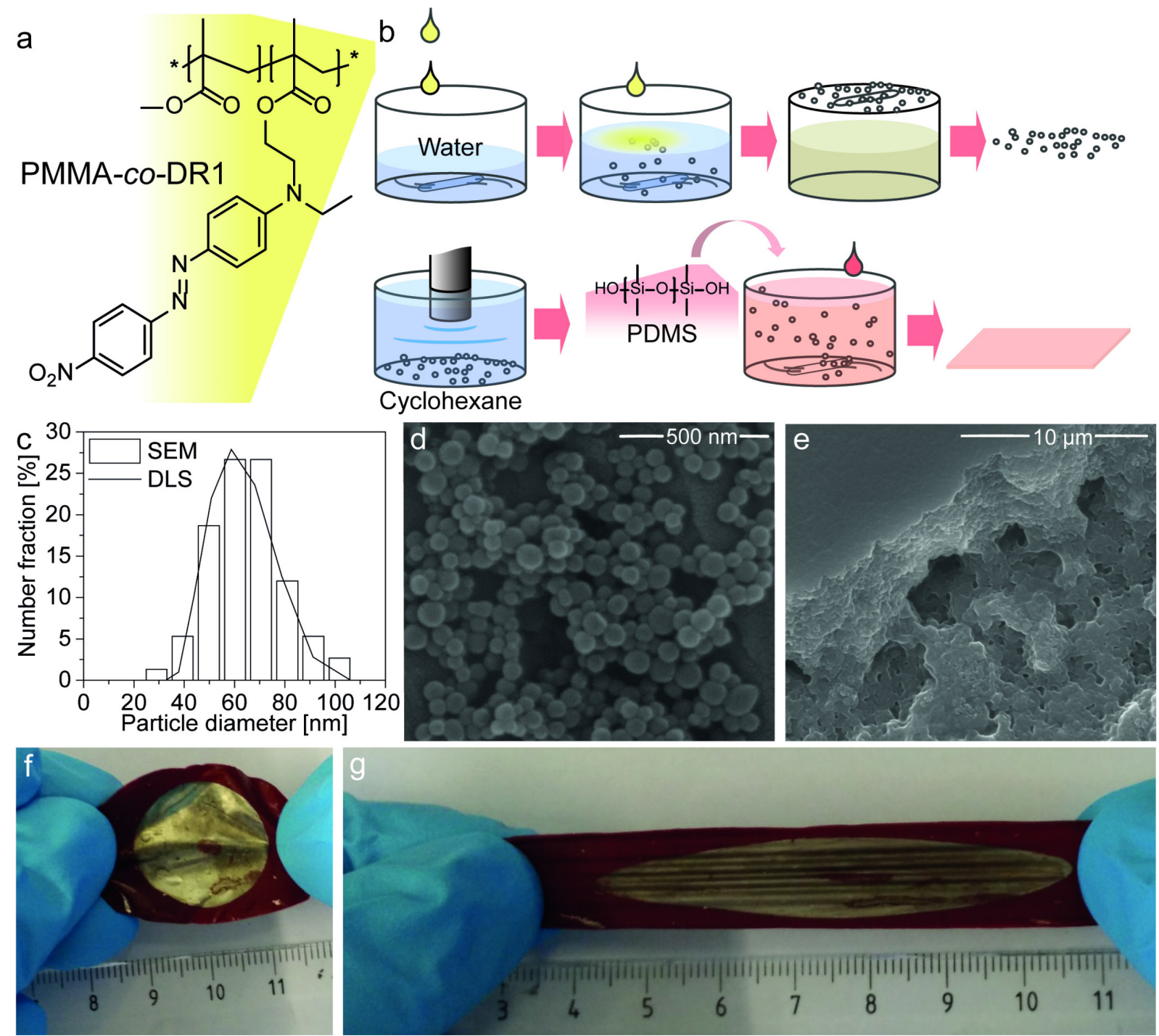

Figure 2. Fabrication of elastic electrets. a) Molecular structure of PMMA-co-DR1MA and b) a schematic representation of it being processed into particles and dispersed into PDMS. c) Size distribution measured by dynamic light scattering (DLS, line) and by counting the particles in. the SEM image (bars) seen in d). e) SEM image of the microstructure of the composite. f-g) Free standing composite film in relaxed and strained condition. The silver paint was applied to illustrate the deformation more clearly and has a diameter of $2 \mathrm{~cm}$.

To produce composites, the particles were first dispersed into cyclohexane via tip sonication while immersed in an ice bath for $20 \mathrm{~min}$. After the particles have been dispersed, hydroxyl- 
terminated PDMS and ethyltriacetoxy silane crosslinker ${ }^{[30]}$ were added to obtain a precomposite solution with a 1:2 weight ratio of particles to PDMS. A homogeneous dispersion was formed after stirring overnight, at which point titanium 2-ethylhexoxide catalyst $(<10 \mu 1$ per g PDMS) was added and films were then produced with the doctor blade method using a Zehntner ZUA 2000. The films were drawn on Teflon coated glass plates. All samples were first dried in air over night to complete the crosslink reaction and then placed in a vacuum oven at $0.1 \mathrm{mbar}$ and $130{ }^{\circ} \mathrm{C}$ for at least $8 \mathrm{~h}$ to remove residual cyclohexane. Some of the film was freeze fractured in liquid nitrogen and the fracture surface examined in SEM. They revealed a homogeneous interior (Figure 2e) with evenly distributed bumps, which are assumed to be particle clusters. The obtained free standing films (Figure $2 \mathbf{f}$ and $\mathbf{g}$ ) were typically $40 \mu \mathrm{m}$ thick.

A number of methods were employed to characterize the dipole relaxation behavior of the copolymer and the composite. Differential scanning calorimetry (DSC) revealed a single glass transition for the copolymer at $124^{\circ} \mathrm{C}$ and the composite at $126^{\circ} \mathrm{C}$ (Figure S2 and S3 in SI), showing that the copolymer phase retained its original $T_{g}$. Because the dipole is immobile in the glassy state, no relaxations should be visible below $T_{g}$. This assumption is confirmed when looking at the impedance spectrum of the materials (Figure 3a and b). The static permittivity $\varepsilon_{s}$ of the copolymer has a value around 5, comparable to PMMA, ${ }^{[31]}$ and dramatically increases to 21 above $120{ }^{\circ} \mathrm{C}$ due to the relaxation of the dipoles. The permittivity of the composite follows a similar trend: At low temperatures it is around 3, and increases to 4 above $120^{\circ} \mathrm{C}$. The less dramatic increase in permittivity is consistent with a sample with diluted dipoles. ${ }^{[32]}$ Additionally, at high temperatures a steep increase in permittivity at low frequencies can be seen in the composite which is attributed to the electrode polarization phenomenon. ${ }^{[33]}$ It indicates mobile charged species in the material. Nonetheless, as will later be shown, this does not hinder the polability of the composite. 
Impedance spectroscopic data are typically analyzed by fitting it with the Havriliak-Negami $(\mathrm{HN})$ equation ${ }^{[34]}$

$\varepsilon^{*}=\varepsilon_{\infty}+i \frac{\sigma}{\varepsilon_{0} \omega}+\sum \frac{\Delta \varepsilon}{\left\{1+\left[i \omega \tau_{n}\right]^{\left.1-\alpha_{n}\right\}^{\beta_{n}}}\right.}$

where $\varepsilon^{*}$ is the relative permittivity, $\varepsilon_{\infty}$ its high frequency limit, $\sigma$ the dc-conductivity, $\omega$ the angular frequency and $\varepsilon_{0}$ the vacuum permittivity. The sum (running over the index $\mathrm{n}$ ) includes polarization contributions from different independent processes. $\Delta \varepsilon_{n}$ and $\tau_{n}$ are the relaxation strengths and characteristic times. The parameters $\alpha_{n}$ and $\beta_{n}$ describe the width and asymmetry of the corresponding dielectric loss peak. It was possible to fit the data from $150{ }^{\circ} \mathrm{C}$ upwards, and the complete set of fit parameters is listed in the IS (Table S3 - S6 in SI). Previously mentioned permittivity values were extracted from the fit parameters. The lines in Figure $3 \mathrm{a}$ and $\mathrm{b}$, representing the $\mathrm{HN}$-fit, demonstrate visually how well the data could be fitted. The relaxation strength is of great help in estimating the possible polarization in a material ${ }^{[35]}$ and the $\mathrm{HN}$-fit makes it possible to extract the relevant values accurately. Typically the polarization is dependent on the strength of the poling field. The copolymer, as

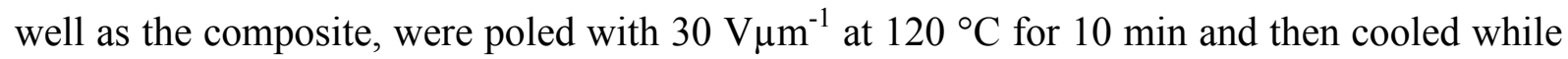
the field was still on. The choice of the poling temperature was guided by studies showing the ideal poling temperature being slightly below $T_{g}{ }^{[23,36]}$ The possible polarization of both materials can be estimated with ${ }^{[37]}$

$P=\varepsilon_{0} \Delta \varepsilon(T) E_{P}$

where $P$ is the polarization, $\varepsilon_{o}$ the vacuum permittivity, $\Delta \varepsilon(T)$ the relaxation strength at the poling temperature and $E_{p}$ the poling field. It must be mentioned, that the $\Delta \varepsilon$ at the poling temperature of $120{ }^{\circ} \mathrm{C}$ should be used in this analysis, but because the measured frequency range was not low enough to resolve the static permittivity at this temperature, the value at $140{ }^{\circ} \mathrm{C}$ was taken as the next best approximation. According to this analysis, polarizations of $4.1 \mathrm{mCm}^{-2}$ and $0.45 \mathrm{mCm}^{-2}$ can be expected for the copolymer and the composite respectively. The actual polarization can be measured with the thermally stimulated depolarization (TSD) 
current method (Figure 3c) and shows that stable values of $3.8 \mathrm{mCm}^{-2}$ and $0.31 \mathrm{mCm}^{-2}$ were achieved. As the poled copolymer has been proven stable after poling, ${ }^{[22]}$ the stability of the composite should be comparable, due to their very similar relaxation behaviours as revealed in the impedance spectra. In fact, the polarization of the composite is initially $0.50 \mathrm{mCm}^{-2}$, higher than anticipated by its relaxation strength, but rapidly decays to $0.31 \mathrm{mCm}^{-2}$ after a few days where it remains stable. PMMA is known to exhibit a prominent heterocharge peak at $120{ }^{\circ} \mathrm{C}$ which disappears after some time, due to interaction with atmospheric molecules, ${ }^{[38]}$ and this addition to the composite TSD current has probably the same origin. Even though heterocharges do not appear in the copolymer TSD response, they could be readily shifted into the PDMS during poling and, being trapped there because PDMS is much less conductive, requiring more time to be eliminated. It is known, that internal charges can contribute to a screening of the piezoelectric current. ${ }^{[39]}$ The fact that the TSD response is on the order of magnitude expected from the calculated polarization based on the actual concentration of dipoles shows that screening by free charges is not a major issue in this material. On the other hand the conductivity of the composite, $10^{-12} \mathrm{~S} \mathrm{~m}^{-1}$ (Figure S4 in SI), is several orders of magnitude higher than in most common electret materials (Teflon, $\sigma=10^{-18} \mathrm{~S} \mathrm{~m}^{-1}$ ). ${ }^{[40]}$ Therefore long term stability of the electret will be undertaken.

After the stability and magnitude of the poled structure has been confirmed with impedance spectroscopy and TSD, the direct piezoelectric coefficient was measured with the Berlincourt method, in which the sample is clamped between two metal discs serving as electrodes and periodically compressed, while charge and force are recorded. The precise description of the measurement setup can be found elsewhere. ${ }^{[41]}$ The composite was poled at different fields by the same method used to pole TSD samples. After determining $d_{33}$ (Figure 3d), the TSD current was measured as the simplest way to confirm the relation between polarization and piezoelectricity. The scatter of the values is relatively high due to the softness of the material. It is nonetheless quite clear, that the $d_{33}$ value changes according to the polarization (Figure 
3e) and the prestress. To begin with, $d_{33}$ decays with the prestress in a seemingly exponential fashion. According to Equation (1), the piezoelectric coefficient is dependent on the elastic modulus of the matrix. Indeed, when the compressive modulus of the composite (Figure 3f) is taken into account, an increasing modulus in the relevant range corresponding to the maximum prestress can be observed, which would explain the lowered $d_{33}$ at high prestresses. It has to be mentioned, that Equation (1) predicts up to 10 times higher $d_{33}$ values than obtained in the experiment even when the sample polarization as measured with TSD is taken into account, but this can be rationalized by non-ideal boundary conditions. The equation assumes a simple serial spring model to calculate the displacement of the charges. In the case of filler concentrations near the percolation threshold, such as here, a stiffer system can be expected. Particles in percolating lines from electrode to electrode would be loaded directly without PDMS acting as buffer. A larger portion of the copolymer would percolate at higher prestresses, changing the presumptions of Equation (1) and eventually lowering the $d_{33}$ to zero. Furthermore, while experimentally the clamped sample resembles the model quite well in that it exhibits no lateral expansion, the model fails to take into account that this kind of clamping translates into a compressive pressure, ${ }^{[42]}$ decreasing the apparent $d_{33}$. Thus Equation (1) should be taken as a qualitative indication of the relationship between polarization and mechanical properties which explains the decreasing $d_{33}$ plausibly. It follows, that a much higher piezoelectric constant can be expected when the sample is operated laterally $\left(d_{31}\right)$, though equally elastic electrodes have to be applied first. 

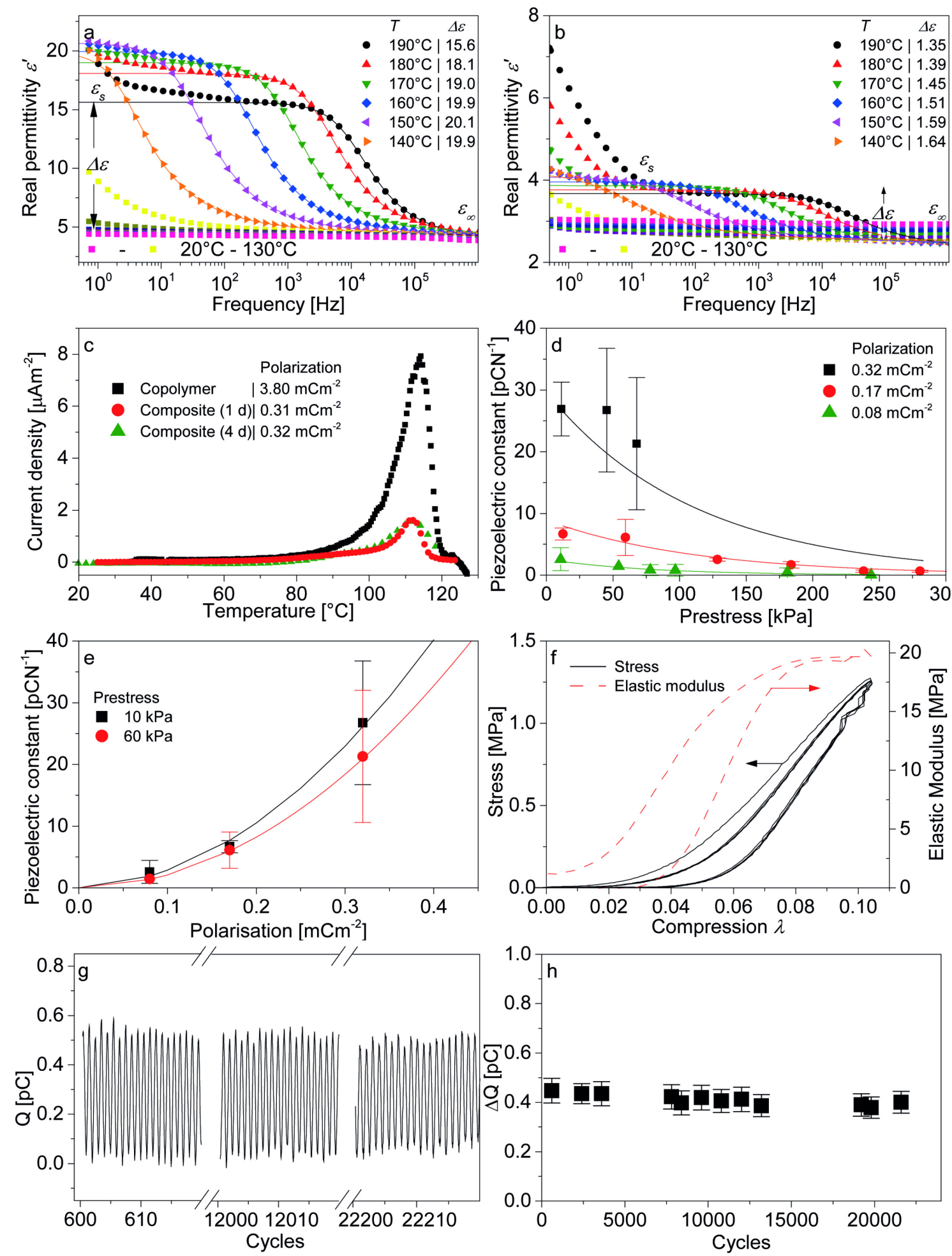

Figure 3. ) Real permittivity of the a) copolymer and b) the composite as measured with TDIS. The measurements are represented by hollow symbols, the HN-fit is represented by the line. The corresponding $\Delta \varepsilon$ is noted next to the temperature. c) TSD current of the copolymer

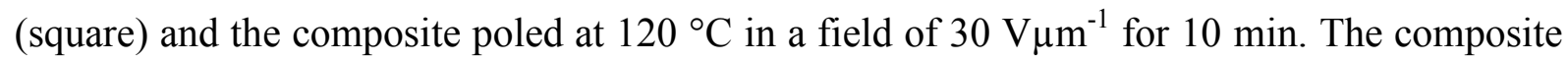
was measured after a day (triangle) and after four days (circle). d) Measured $d_{33}$ of the 
composite with different polarizations plotted against increasing prestress (symbols), and an exponential decay fit assuming similar decay constants (line). e) The dependence of the amplitude of $d_{33}$ on polarization (symbols) and the fit (line). f) Compressive tensile curve of the composite plotted as engineering stress $(\sigma)$ vs compression $(\lambda)$, repeated four times and its derivative elastic modulus $(Y)$ g) Charge response and h) the average peak to peak charge difference during a cyclic load test at selected intervals.

Regarding the polarization dependence, the $d_{33}$ values increase from an amplitude of $2.5 \mathrm{pCN}^{-1}$ to $26.7 \mathrm{pCN}^{-1}$ in a seemingly quadratic relationship to the polarization. Though this dependence is debatable due to the high noise in the measurement, the increasing value of $d_{33}$ with higher polarizations, leads to the indisputable conclusion, that the polarization is responsible for the piezoelectric response. The relaxation of the composite under cyclic load was considered in an experiment, where the charge response over more than 20000 load cycles at $10 \mathrm{~Hz}$ and $80 \mathrm{mN}$ amplitude was recorded at selected intervals (Figure $\mathbf{3 g}$ and $\mathbf{h}$ ). No significant decrease in the collected charge was detected over this time period. This is in line with the TSD findings where the polarization of the composite remains stable after several days. This also indicates that relaxations of whole poled particles are not likely in the present composite. The $d_{33}$ values are comparable to what is commonly obtained in PVDF, ${ }^{[43]}$ but has to be viewed in light of the elastomeric nature of the material. In the current iteration, the composite has an elastic modulus of $10 \mathrm{MPa}$, two orders of magnitude lower than PVDF, and a strain at break of $300 \%$ (Figure S5 in SI).

The electret could be used in sensors, though the prestress dependent $d_{33}$ would limit the electret to simple on-off applications. It is interesting to note, that the mechanical properties of the current material bears similarities to natural skin ${ }^{[44]}$ which would recommend it to biomedical applications. It is also important to keep in mind that the properties can be varied considerably by changing the composition. Due to the NLO-nature of the filler, optical applications can be considered, though feasibility studies have first to be conducted, for 
example with regards to diffuse scattering at the particle matrix interphase. More practically, the organic nature of the composite allows for cost efficient method to produce a variety of materials which are also more straightforward to dispose of. The thermal properties of organics are also much more easily tailored to specific needs, though normally a high $T_{g}$ is most desired.

In conclusion, an all-organic, soft and truly elastomeric material with competitive piezoelectric properties was demonstrated. The universal materials design is based on a silicone composite incorporating polable submicron filler particles that were prepared by nanoprecipitation of a NLO copolymer (PMMA-co-DR1MA). After poling, piezoelectric coefficients $d_{33}$ of $26.7 \mathrm{pCN}^{-1}$ was obtained, demonstrating piezoelectric performance comparable to the best ferroelectric polymers. Investigations are currently underway to ascertain the thermal long term stability, as well as the efficiency in lateral operation $\left(d_{31}\right)$ of the composite. Due to the plethora of NLO materials ${ }^{[45]}$ which can be combined with elastomeric matrices to produce composites with tailor-made electromechanical properties, a number of possible applications can be envisaged where soft piezoelectric materials are required. They range from components in soft electronics and energy harvesting applications to stretchable transducers.

\section{Experimental}

Please see the Supporting Information.

\section{Acknowledgements}

We gratefully acknowledge the Swiss National Science Foundation (200021L_140577/1) and the Swiss Federal Laboratories for Materials Science and Technology Empa for financial support. We are also thankful of the help of T. D'Anna in producing the nanoparticles. Furthermore, we would like to thank Dr. R. Brönnimann for his kind support with the electric measurements, B. Fischer for the DSC measurements, and Dr. F. Clemens for his support and insightful discussions (all Empa). We are also indebted to Prof. S. Bauer from the University of Linz for his useful input.

\section{Author Contributions}

Y.S.K. performed the synthesis and the characterization of all materials and wrote the manuscript together with D.M.O with input from all authors. D.M.O. initiated the activity for elastic polable composites, designed the materials, and coordinated this research. F.A.N. and 
D.D. helped with the interpretation of the results. All authors have given approval to the final version of the manuscript.

[1] B. Gross, Charge Storage in Solid Dielectrics, Elsevier, Amsterdam, 1964.

[2] H. Kawai, Japan Soc. Appl. Phys. 1969, 8, 975.

[3] R. Gerhard-Multhaupt, IEEE Trans. Electr. Insul. 1987, 22, 531.

[4] B. P. Mahale, D. Bodas, S. A. Gangal, 2011 6th IEEE Int. Conf. Nano/Micro Eng. Mol. Syst. 2011, 658-661.

[5] F. S. Foster, K. A. Harasiewicz, M. D. Sherar, IEEE Trans. Ultrason. Ferroelectr. Freq. Control 2000, 47, 1363.

[6] H. R. Gallantree, IEE Proc. I Solid State Electron Devices 1983, 130, 219.

[7] D. K. Das-Gupta, Ferroelectrics 1991, 118, 165.

[8] K. C. Kao, in Dielectric Phenomena in Solids, Elsevier, Amsterdam, 2004, Ch. 5.

[9] S. Bauer, J. Appl. Phys. 1996, 80, 5531.

[10] W. Eisenmenger, M. Haardt, Solid State Commun. 1982, 41, 917.

[11] D. Rollik, S. Bauer, R. Gerhard-Multhaupt, J. Appl. Phys. 1999, 85, 3282.

[12] M. G. Broadhurst, G. T. Davis, in Electrets, Springer, Berlin, 1980, Ch. 5.

[13] P. N. Prasad, B. A. Reinhardt, Chem. Mater. 1990, 2, 660.

[14] W. Ren, S. Bauer, S. Yilmaz, W. Wirges, R. Gerhard-Multhaupt, J. Appl. Phys. 1994, $75,7211$.

[15] T. Q. Trung, N.-E. Lee, Adv. Mater. 2016, 28, 4338.

[16] B. C.-K. Tee, A. Chortos, A. Berndt, A. K. Nguyen, A. Tom, A. McGuire, Z. C. Lin, K. Tien, W.-G. Bae, H. Wang, P. Mei, H.-H. Chou, B. Cui, K. Deisseroth, T. N. Ng, Z. Bao, Science 2015, 350, 313.

[17] X. Li, R. Zhang, W. Yu, K. Wang, J. Wei, D. Wu, A. Cao, Z. Li, Y. Cheng, Q. Zheng, 
R. S. Ruoff, H. Zhu, Sci. Rep. 2012, 2, 870.

[18] S. Park, H. Kim, M. Vosgueritchian, S. Cheon, H. Kim, J. H. Koo, T. R. Kim, S. Lee, G. Schwartz, H. Chang, Z. Bao, Adv. Mater. 2014, 26, 7324.

[19] R. Gerhard, Proc. SPIE, 2016, 9798, 97980T-1.

[20] S. Schubert, J. T. Delaney, Jr, U. S. Schubert, Soft Matter 2011, 7, 1581.

[21] D. Lei, J. Runt, A. Safari, R. E. Newnham, Macromolecules 1987, 20, 1797.

[22] A. Nahata, J. Shan, J. T. Yardley, C. Wu, J. Opt. Soc. Am. B 1993, 10, 1553.

[23] D. M. Burland, R. D. Miller, C. A. Walsh, Chem. Rev. 1994, 94, 31.

[24] S. Bauer, S. Bauer-Gogonea, B. Ploss, B. Ploss, J. Non. Cryst. Solids 2005, 351, 2759.

[25] C. S. Kang, H.-J. Winkelhahn, M. Schulze, D. Neher, G. Wegner, Chem. Mater. 1994, $6,2159$.

[26] X. Mo, K. Kinemura, T. Yamada, A. Otomo, D. Taguchi, T. Manaka, M. Iwamoto, Jpn. J. Appl. Phys. 2014, 53, 1.

[27] N. Li, J. Lu, X. Xia, Q. Xu, L. Wang, Polymer 2009, 50, 428.

[28] E. Lepeltier, C. Bourgaux, P. Couvreur, Adv. Drug Deliv. Rev. 2014, 71, 86.

[29] International Organization for Standardization, Particle Size Analysis - Dynamic Light Scattering (DLS), ISO 22412:2008, Geneva, 2008.

[30] D. M. Opris, M. Molberg, C. Walder, Y. S. Ko, B. Fischer, F. a. Nüesch, Adv. Funct. Mater. 2011, 21, 3531.

[31] W. M. Haynes, in CRC Handbook of Chemistry and Physics, Taylor \& Francis, New York, 2015, Ch. 13.

[32] F. Kremer, A. Schönhals, in Broadband Dielectric Spectroscopy, Springer, Berlin, 2003, Ch. 6.

[33] P. Ben Ishai, M. S. Talary, A. Caduff, E. Levy, Y. Feldman, Meas. Sci. Technol. 2013, $24,102001$.

[34] S. Havriliak, S. Negami, Polymer 1967, 8, 161. 
[35] W. Kohler, D. R. Robello, P. T. Dao, C. S. Willand, R. S. Williams, J Chem Phys 1990, 93, 9157.

[36] F. I. Mopsik, M. G. Broadhurst, J. Appl. Phys. 1975, 46, 4204.

[37] L. Onsager, J. Am. Chem. Soc. 1936, 58, 1486.

[38] J. Vanderschueren, J. Polym. Sci. Polym. Phys. Ed. 1974, 12, 991.

[39] J. Briscoe, S. Dunn, Nano Energy, 2015, 14, 15.

[40] J. Malecki, Int. Symp. Electrets 1996, 9, 110.

[41] A. Barzegar, D. Damjanovic, N. Setter, IEEE Trans. Ultrason. Ferroelectr. Freq. Control 2004, 51, 262.

[42] K. Lefki, G. Dormans, J. Appl. Phys. 1994, 76, 1764.

[43] K. S. Ramadan, D. Sameoto, S. Evoy, Smart Mater. Struct. 2014, 23, 033001.

[44] J. L. Gennisson, T. Baldeweck, M. Tanter, S. Catheline, M. Fink, L. Sandrin, C.

Cornillon, B. Querleux, IEEE Trans. Ultrason. Ferroelectr. Freq. Control 2004, 51, 980.

[45] L. R. Dalton, P. A. Sullivan, D. H. Bale, Chem. Rev. 2010, 110, 25.

\section{The table of contents}

Copolymer nanoparticles with a highly polar repeating unit are blended with an elastic matrix and poled at elevated temperatures. The composite exhibits piezoelectricity due to the overall polarization imparted by the particles which can be easily modulated thanks to the soft matrix.

Keywords: Electret, PDMS, Disperse Red 1, Piezoelectric, Elastomer

Y.S. Ko, F. A. Nüesch, D. Damjanovic and D. M. Opris*

An All-Organic Elastomeric Electret Composite 


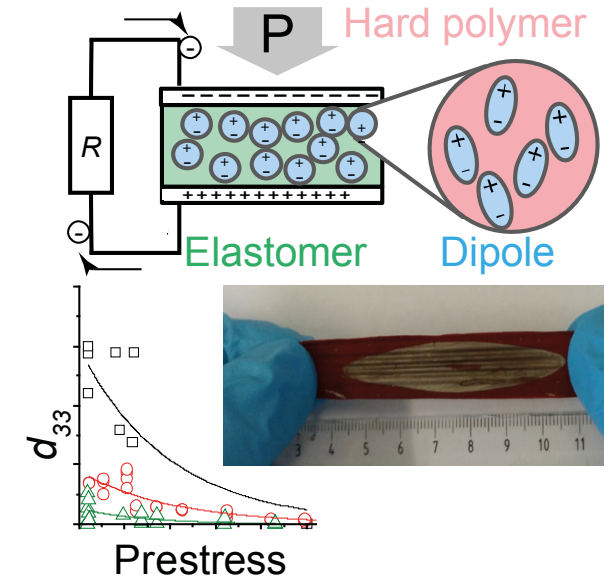

